\title{
Improved error thresholds for measurement-free error correction
}

\author{
Daniel Crow, Robert Joynt, and M. Saffman \\ Department of Physics, University of Wisconsin-Madison, \\ 1150 University Avenue, Madison, Wisconsin 53706
}

(Dated: October 1, 2018)

\begin{abstract}
Motivated by limitations and capabilities of neutral atom qubits, we examine whether measurement-free error correction can produce practical error thresholds. We show that this can be achieved by extracting redundant syndrome information, giving our procedure extra fault tolerance and eliminating the need for ancilla verification. The procedure is particularly favorable when multi-qubit gates are available for the correction step. Simulations of the bit-flip, Bacon-Shor, and Steane codes indicate that coherent error correction can produce threshold error rates that are on the order of $10^{-3}$ to $10^{-4}$ - comparable with or better than measurement-based values, and much better than previous results for other coherent error correction schemes. This indicates that coherent error correction is worthy of serious consideration for achieving protected logical qubits.
\end{abstract}

PACS numbers: 03.67.Pp,32.80.-t,32.80.Qk

An important near-term goal in quantum information processing is the construction and operation of a highquality logical qubit. This goal is currently being pursued in several physical systems [1 4]. One promising candidate system is an array of neutral atoms held in optical or magnetic traps [5, 6]. The quantum information is stored in atomic hyperfine clock states. This system has several attractive features: each natural qubit is identical, clock states exhibit long coherence times measured in seconds, and state preparation and state measurement can be performed on msec timescales using well-developed techniques of optical pumping and detection of resonance fluorescence [7, 8]. Arrays of individually addressable neutral atom qubits have been demonstrated in 1D [9, 10], 2D [11 14], and 3D [15]. Qubit numbers of order 100 have been demonstrated in 2D and $3 \mathrm{D}$ and in principle these numbers could be extended to several thousands using available technology. Lastly, the available gate set is universal, based on microwave and laser light for single qubit rotations together with Rydberg state mediated interactions for two-qubit, and multi-qubit, entangling gates [6].

Achieving logical protection requires an error correction procedure compatible with available operations. Standard error correction protocols rely on performing frequent syndrome measurements [16, 17]. This turns out not to be well suited for neutral atom implementations for two reasons. First, the time needed for state measurements is currently several orders of magnitude longer than for gate operations. Second, it is difficult to measure a single atomic qubit in an array without scattered light corrupting the state of nearby qubits, although a number of possible solutions to this problem are under study [18].

These challenges motivate the consideration of coherent, or measurement-free, error correction (CEC) methods 1922 . Like standard measurement-based error correction (MEC) [1 4], techniques for measurement-free er- ror correction are based on stabilizer codes. However, there has been strong skepticism that CEC can produce error thresholds close to those of MEC [23 25], though Paz-Silva et al. did achieve a CEC threshold only about one order of magnitude worse than MEC [20]. We improve this result by nearly 2 orders of magnitude by taking advantage of the resources available to neutral atoms, in combination with a novel syndrome extraction technique.

CEC is particularly attractive for neutral atom and trapped ion approaches that rely on light scattering for entropy removal. As part of an error correction cycle, entropy in the data qubits is transferred to fresh ancilla qubits, and is subsequently removed by resetting the ancillas. Although an ancilla reset requires optical pumping and light scattering, the number of scattered photons is typically 1-2 orders of magnitude less than would be needed for state measurement in MEC.

CEC can additionally benefit from an additional resource of neutral atom systems, since the computational capabilities include native Toffoli and $\mathrm{C}_{k} \mathrm{NOT}$ gates. These $\mathrm{C}_{k}$ NOTs can potentially achieve fidelities as high as $90 \%$ for $k \sim 35$, while for smaller $k$ the fidelities of the native gates are expected to beat fidelities of the decompositions into 1- and 2-qubit gates [26, 27]. Similarly, Rydberg interactions allow for parallel CNOT gates in which a single control qubit targets multiple qubits simultaneously, improving the time required for syndrome extraction. Native Toffoli gates have also been demonstrated using trapped ion [28] and superconducting qubits [29]; thus the techniques presented here could potentially be adapted to other platforms.

A quantum error correction code is determined by the number of physical qubits and by the stabilizing group that fixes the logical subspace. This stabilizer group, with elements $S_{i}$, is determined by its generators. Given $n$ stabilizer generators, we can consider $2^{n}-1$ distinct non-trivial products of the generators, forming additional 
TABLE I. Correctly extracted syndromes for single-qubit bitflip errors on the logical $|000\rangle$ state. The table is easily extended to errors on the $|111\rangle$ state.

\begin{tabular}{ccccc}
\hline \hline & $|000\rangle$ & $|100\rangle$ & $|010\rangle$ & $|001\rangle$ \\
\hline$Z_{1} Z_{2}$ & 0 & 1 & 1 & 0 \\
$Z_{2} Z_{3}$ & 0 & 0 & 1 & 1 \\
$Z_{1} Z_{3}$ & 0 & 1 & 0 & 1 \\
\hline \hline
\end{tabular}

stabilizers. If stabilizer values could be extracted and processed without error, only the stabilizer generators need to be measured, and additional stabilizers would not provide additional useful information. The procedure we propose is to copy onto ancillas the redundant information of a subset of these additional stabilizers. This enables one not only to identify data errors, but also errors that occur during syndrome extraction. The redundancy becomes useful when combined with the Toffoli and $\mathrm{C}_{k}$ NOT gates, where the quantum gates act as logical 'AND' gates to ensure that stabilizer values agree, conditionally targeting errors only if extracted stabilizer values match expected syndromes. Using this method, the ancilla qubits store only classical information-i.e., they are immune to phase errors and are not directly entangled with each other. We discuss this approach for three codes: 3-qubit bit-flip, 9-qubit Bacon-Shor, and 7qubit Steane.

The 3-qubit bit-flip (BF) code has logical states $|0\rangle_{L}=$ $|000\rangle$ and $|1\rangle_{L}=|111\rangle$ with the usual stabilizers

$$
S_{1}=Z_{1} Z_{2} ; \quad S_{2}=Z_{2} Z_{3} .
$$

The values of $S_{1}$ and $S_{2}$ correctly identify single-qubit errors, and each syndrome value corresponds to a distinct correction procedure. Thus, any extraction errors leading to an incorrect value of either stabilizer leads to an incorrect procedure, likely resulting in a logical error. However, by considering the additional stabilizer

$$
S_{3}=S_{1} S_{2}=Z_{1} Z_{3}
$$

it is possible to correctly identify if a single error occurs during ancilla preparation or syndrome extraction (collectively: extraction errors). This property follows from the fact that a correctly extracted syndrome always produces an even number of ancillas in the logical $|1\rangle$ state, as shown in Table \. Therefore, a single extraction error occurs if an odd number of ancilla qubits occupy a logical $|1\rangle$ state. The error-correction circuit is shown in Fig. 1 The circuit makes use of $\mathrm{C}_{3} \mathrm{NOT}$ gates, to correct errors on the data qubits only if the ancillary state corresponds to a valid syndrome.

An advantage of using additional stabilizer information is that the procedure does not require separate an-

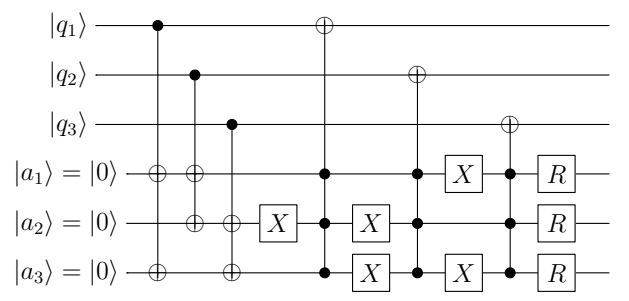

FIG. 1. The full measurement-free extraction and correction circuit for the $\mathrm{BF}$ code. The first 3 gates are for syndrome extraction. The combination of $X$ gates and $\mathrm{C}_{3} \mathrm{NOT}$ gates detect properly extracted syndromes and correct errors accordingly. If a syndrome value is incorrectly extracted, the data qubits are not affected. Reset operations are performed in the final step, indicated with $R$ operations. This circuit also demonstrates the bit-flip correction procedure for the BS code, taking each $\left|q_{i}\right\rangle$ to be a row in the BS code. Then each CNOT gate is interpreted as 3 CNOT gates, one controlled by each qubit in the row. The $\mathrm{C}_{3} \mathrm{NOT}$ gates target any single qubit in the row. A similar procedure is required for phase errors in the BS code.

cilla verification. That is, single-qubit extraction errors can be detected simply from the combinatorics of properly extracted syndromes. In our CEC circuits, the $\mathrm{C}_{k} \mathrm{NOT}$ gates act nontrivially on data qubits - i.e. correct errors - only if syndromes are properly extracted. This implies that pre-existing data errors can survive a faulty CEC cycle. However, with high probability the surviving data error is simply corrected during the following cycle.

The 9-qubit Bacon-Shor (BS) code is obtained by layering the bit-flip code, with one layer designed to protect against phase errors, resulting in a code that can correct arbitrary single-qubit errors. The logical $X$ and $Z$ operators are just $X^{\otimes 9}$ and $Z^{\otimes 9}$, respectively. The error correction procedure is quite similar to the bit-flip code, still needing just 3 ancillas. Due to the underlying symmetry, this code requires only 4 stabilizer generators. With the data qubits in a $3 \times 3$ grid, the stabilizers are:

$$
\begin{array}{ll}
Z_{U}=\left(\begin{array}{lll}
Z & Z & Z \\
Z & Z & Z \\
I & I & I
\end{array}\right), Z_{D}=\left(\begin{array}{lll}
I & I & I \\
Z & Z & Z \\
Z & Z & Z
\end{array}\right), \\
X_{L}=\left(\begin{array}{lll}
X & X & I \\
X & X & I \\
X & X & I
\end{array}\right), X_{R}=\left(\begin{array}{lll}
I & X & X \\
I & X & X \\
I & X & X
\end{array}\right) .
\end{array}
$$

The procedure to perform error correction then proceeds in a manner similar to the BF code. To correct bitflip errors, we consider the additional stabilizer $Z_{U} Z_{D}$. The circuit then proceeds as in Fig. 1 but now taking each $\left|q_{i}\right\rangle$ to correspond to a single row of 3 data qubits. Each CNOT gate in the circuit can then be interpreted as 3 physical CNOT gates - one for each data qubit. The $\mathrm{C}_{k}$ NOT gates can target any single physical qubit in the row. The procedure for correcting phase errors is analogous, although extraction and correction is done by col- 
umn. Additional information is provided in the supplementary material.

The 7-qubit Steane code has 6 stabilizer generators and requires 7 ancillas to correct arbitrary single-qubit errors. Three $Z$-type stabilizers

$$
S_{1}^{Z}=Z_{1} Z_{2} Z_{3} Z_{7} ; \quad S_{2}^{Z}=Z_{1} Z_{2} Z_{4} Z_{6} ; \quad S_{3}^{Z}=Z_{1} Z_{3} Z_{4} Z_{5}
$$

detect bit-flip errors, while $X$-type stabilizers detect phase-flip errors and are obtained from the $Z$-type operators by replacing each $Z_{i}$ with $X_{i}$. The logical operators are $Z_{L}=Z^{\otimes 7}$ and $X_{L}=X^{\otimes 7}$

We will restrict our discussion to bit-flip errors; phase errors follow analogously. With $3 Z$-type generators, we can form 7 distinct stabilizers. Then error correction proceeds as follows: (1) extract the 7 stabilizer values onto 7 ancilla qubits, and (2) use a sequence of $7 \mathrm{C}_{4} \mathrm{NOT}$ gates to correct errors, matching each target data qubit $q_{i}$ to the unique set of control ancilla qubits whose corresponding stabilizers act on $q_{i}$. The details of this procedure are discussed in the supplementary material, and the circuit is shown in Fig. 2.

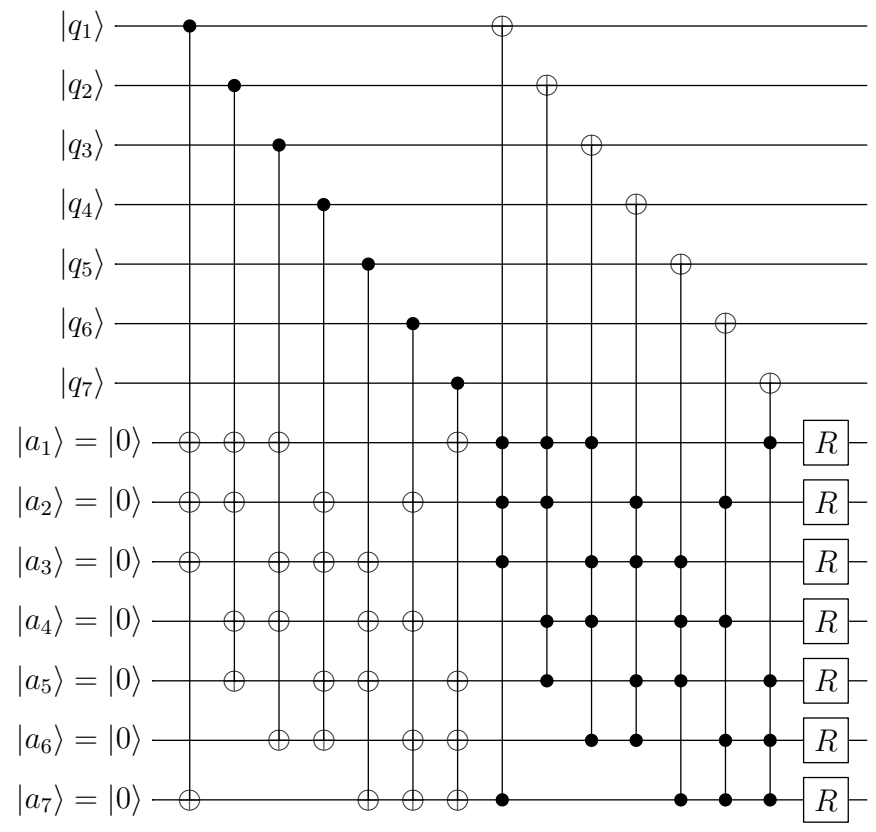

FIG. 2. Error correction circuit for the Steane code for bit-flip errors. The circuit for phase errors is similar.

We performed a numerical simulation of measurementfree error correction using the circuits shown in the previous section. We adopted an error model controlled by two error-rate parameters: the gate rate $p_{\text {gate, }}$ and the memory (or idle-gate) rate $p_{\text {mem }}$. All single-qubit gate errors are all assumed to be depolarizing, i.e. if an error occurs on qubit $i$, then a single-qubit Pauli is selected at random and applied to qubit $i$. Two-qubit gate errors occur with the same probability $p_{\text {gate }}$ as single-qubit
TABLE II. Comparison of CEC and MEC gate thresholds. The BS and Steane MEC values are the best values obtained for each code in Ref. [33], while the BF value is obtained from [34] and is scaled by 1.5 since our error rate includes phase errors.

\begin{tabular}{lccc}
\hline \hline & $\mathrm{BF}$ & $\mathrm{BS}$ & Steane \\
\hline CEC $p_{\text {mem }}=0$ & 0.010 & $1.8 \times 10^{-3}$ & $8.9 \times 10^{-5}$ \\
CEC $p_{\text {mem }}=p_{\text {gate }}$ & $5.5 \times 10^{-4}$ & $1.01 \times 10^{-4}$ & $3.2 \times 10^{-5}$ \\
MEC $p_{\text {mem }}=0$ & & $2.6 \times 10^{-4}$ & $5 \times 10^{-4}$ \\
MEC $p_{\text {mem }}=p_{\text {gate }}$ & $\sim 0.03$ & $2.1 \times 10^{-4}$ & $2.6 \times 10^{-4}$ \\
\hline \hline
\end{tabular}

gates, but the error is chosen at random from the set of 2qubit Paulis. For multi-qubit gates, each control-target pair of qubits is treated as a two-qubit gate site, subject to error model as other two qubit errors. The effect is that $\mathrm{C}_{k} \mathrm{NOT}$ gates have an error rate of roughly $k \cdot p_{\text {gate }}$, roughly matching physical error models [27].

The simulated circuits required the ability to perform single-qubit Pauli, CNOT, and $\mathrm{C}_{k} \mathrm{NOT}$ gates. The state evolution was performed using stabilizer simulation, in a manner similar to the techniques outlined by Aaronson and Gottesman in [30]. However, the $\mathrm{C}_{k} \mathrm{NOT}$ gate is not in the Clifford group, and is not typically simulable in an efficient manner. However, in every circuit studied here, the $\mathrm{C}_{k}$ NOT gates are always controlled by the ancilla qubits, which only store classical information and are modeled as classical bits.

To efficiently collect data on the circuit, we used simulation and computational techniques similar to those in Refs. 31] and [32], with additional detail in the supplementary material. Using these techniques, we can easily and accurately estimate logical error rates. In principle, these methods could be scaled to more qubits and additional input parameters in a straightforward manner.

The threshold was evaluated by determining $p_{\text {gate }}$ such that the logical error rate $p_{\log }$ satisfied $p_{\log }\left(p_{\text {gate }}\right)=p_{\text {gate }}$. To reduce $p_{\log }$ to a function of a single parameter, we set $p_{\text {mem }}$ to a fixed value, or set $p_{\text {mem }}=p_{\text {gate }}$. For neutral atom qubits, memory error rates are one to two orders of magnitude below gate rates. In this region of parameter space, varying $p_{\text {mem }}$ had little effect on the threshold gate rate, demonstrated in Figs. 3 and 4 . The threshold results are summarized in Table II

The difference between the thresholds for the BaconShor and Steane codes highlights the behavior of $\mathrm{C}_{k} \mathrm{NOT}$ gates with unprotected ancilla qubits. In the Steane code, the successful correction of a data error depends on the successful extraction of 4 syndrome values, while the Bacon-Shor depends on only 3 syndrome values. Furthermore, the syndrome extraction process for the Steane code requires 56 CNOT gates, compared with 36 for the Bacon-Shor code. Thus, the $\mathrm{C}_{k} \mathrm{NOT}$ gates performing 


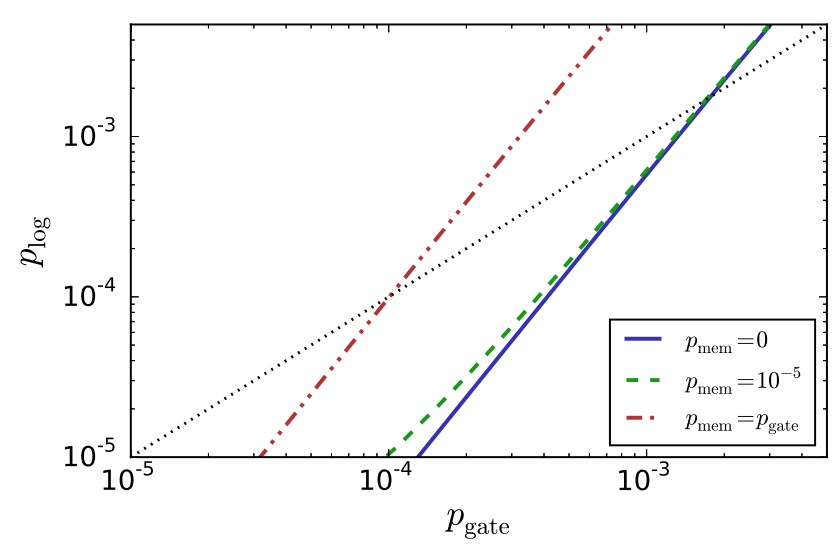

FIG. 3. (Color online) Logical error rate vs. gate error rate for the Bacon-Shor code, with three different choices of memory error rate. The dotted line shows $p_{\log }=p_{\text {gate }}$. The difference between the curves with memory rates of 0 and $10^{-5}$ is minimal.

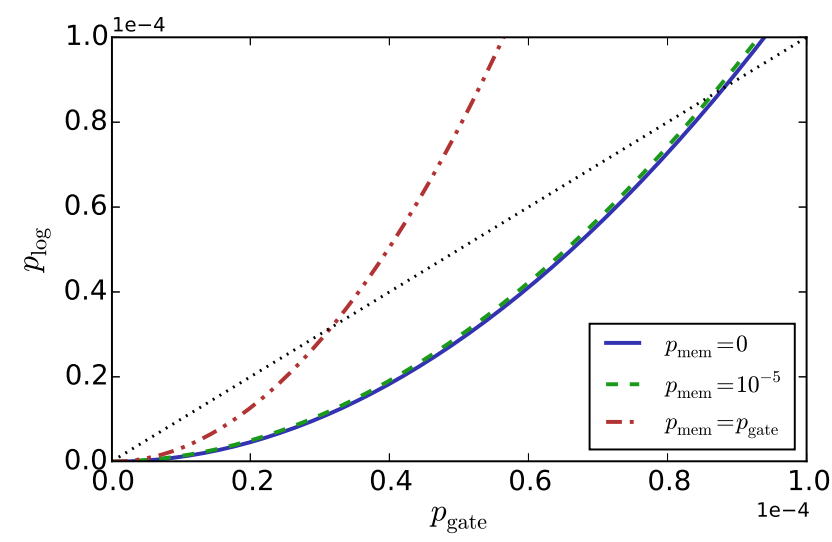

FIG. 4. (Color online) Logical error rate vs. gate error rate for the Steane code, with three different choices of memory error rate. The dotted line shows $p_{\log }=p_{\text {gate }}$. Note the near overlap between the curves with memory rates of 0 and $10^{-5}$.

error correction are significantly more likely to fail in the case of the Steane code. However, in all procedures studied here, failures in extraction do not propagate new errors onto data qubits.

Earlier work on measurement free error correction found a threshold of $p_{T} \approx 3.8 \times 10^{-5}$ for the 9-qubit Bacon-Shor code [20]. Thus, our work indicates a substantial improvement over this value. Additionally, the earlier value needs 18 additional ancilla qubits, while our protocol needs just 3 . The differences can be attributed to the combination of extracting additional stabilizer values, coupled with the efficiency of $\mathrm{C}_{k} \mathrm{NOT}$ gates for performing classical logic.

Directly comparing our result to measurement-based results is not straightforward - measurement-based values depend on the chosen ancilla verification scheme and do not use extra stabilizer information. In addition, there is some arbitrariness in our choice of an error model for $\mathrm{C}_{k}$ NOT gates. With these caveats, in Table II we compare our results to the best measurement-based threshold values from Ref. 33], which are also first-level depolarizing thresholds. The dramatic difference in thresholds for the case of the Bacon-Shor seems to exist only in the regime where memory error rates are small. In this regime, errors are dominated by gate errors, but the circuit lengths for CEC using neutral atom resources are typically quite small - and certainly smaller than those required for ancilla verification. Without the efficiency of multiqubit resources, we would expect thresholds to drop.

Somewhat surprisingly, the thresholds calculated for CEC are comparable to, and, in the case of Bacon-Shor, better than thresholds calculated for MEC. The threshold error rates are encouraging - the bit-flip and BaconShor codes both yield values that are realistic for neutral atom systems [35]. The overhead required for CEC is not greater, and possibly even less than that in MEC, though $\mathrm{C}_{k} \mathrm{NOT}$ gates are required. Furthermore, the technique of using redundant syndrome extraction can potentially be useful in other architectures. Certainly, measurement problems are not restricted to neutral atoms. Furthermore, redundant syndrome extraction can potentially be used in MEC to avoid ancilla verification, which we plan to explore in future research.

The simulations were performed using the University of Wisconsin Center for High Throughput Computing. We gratefully acknowledge the support of the staff. MS acknowledges support from the IARPA MQCO program through ARO contract W911NF-10-1-0347 and the ARL-CDQI through cooperative agreement W911NF-152-0061. We also thank G. A. Paz-Silva, S. N. Coppersmith, M. Friesen, J. Ghosh, and E. Ercan for helpful discussions and communications.

[1] P. Schindler, J. T. Barreiro, T. Monz, V. Nebendahl, D. Nigg, M. Chwalla, M. Hennrich, and R. Blatt, Science 332, 1059 (2011)

[2] G. Waldherr, Y. Wang, S. Zaiser, M. Jamali, T. Schulte-Herbruggen, H. Abe, T. Ohshima, J. Isoya, J. F. Du, P. Neumann, and J. Wrachtrup, Nature 506, 204 (2014)

[3] J. Kelly, R. Barends, A. G. Fowler, A. Megrant, E. Jeffrey, T. C. White, D. Sank, J. Y. Mutus, B. Campbell, Y. Chen, Z. Chen, B. Chiaro, A. Dunsworth, I. C. Hoi, C. Neill, P. J. J. O/'Malley, C. Quintana, P. Roushan, A. Vainsencher, J. Wenner, A. N. Cleland, and J. M. Martinis, Nature 519, 66 (2015).

[4] D. Riste, S. Poletto, M. Z. Huang, A. Bruno, V. Vesterinen, O. P. Saira, and L. DiCarlo, Nat. Comm. 6 (2015)

[5] T. D. Ladd, F. Jelezko, R. Laflamme, Y. Nakamura, C. Monroe, and J. L. O'Brien, Nature 464, 45 (2010). 
[6] M. Saffman, T. G. Walker, and K. Mølmer, Rev. Mod. Phys. 82, 2313 (2010).

[7] A. Fuhrmanek, R. Bourgain, Y. R. P. Sortais, and A. Browaeys, Phys. Rev. Lett. 106, 133003 (2011)

[8] M. J. Gibbons, C. D. Hamley, C.-Y. Shih, and M. S. Chapman, Phys. Rev. Lett. 106, 133002 (2011).

[9] D. Schrader, I. Dotsenko, M. Khudaverdyan, Y. Miroshnychenko, A. Rauschenbeutel, and D. Meschede, Phys. Rev. Lett. 93, 150501 (2004).

[10] C. Knoernschild, X. L. Zhang, L. Isenhower, A. T. Gill, F. P. Lu, M. Saffman, and J. Kim, Appl. Phys. Lett. 97, 134101 (2010)

[11] T. Xia, M. Lichtman, K. Maller, A. W. Carr, M. J. Piotrowicz, L. Isenhower, and M. Saffman, Phys. Rev. Lett. 114, 100503 (2015).

[12] H. Labuhn, S. Ravets, D. Barredo, L. Béguin, F. Nogrette, T. Lahaye, and A. Browaeys, Phys. Rev. A 90, 023415 (2014).

[13] C. Weitenberg, S. Kuhr, K. Mølmer, and J. F. Sherson, Phys. Rev. A 84, 032322 (2011)

[14] M. Schlosser, S. Tichelmann, J. Kruse, and G. Birkl, Quantum Information Processing 10, 907 (2011).

[15] Y. Wang, X. Zhang, T. A. Corcovilos, A. Kumar, and D. S. Weiss, Phys. Rev. Lett. 115, 043003 (2015)

[16] M. A. Nielsen and I. L. Chuang, Quantum Computation and Quantum Information (Cambridge U.P., 2010).

[17] A. G. Fowler, M. Mariantoni, J. M. Martinis, and A. N. Cleland, Phys. Rev. A 86, 032324 (2012).

[18] I. I. Beterov and M. Saffman, Phys. Rev. A 92, 042710 (2015).

[19] V. Nebendahl, H. Häffner, and C. F. Roos, Phys. Rev. A 79, 012312 (2009).
[20] G. A. Paz-Silva, G. K. Brennen, and J. Twamley, Phys. Rev. Lett. 105, 100501 (2010)

[21] C.-K. Li, M. Nakahara, Y.-T. Poon, N.-S. Sze, and H. Tomita, Quantum Info. Comput. 12, 149 (2012)

[22] V. Nebendahl, Phys. Rev. A 91, 022332 (2015)

[23] D. Aharonov and M. Ben-Or, SIAM Journal on Computing 38, 1207 (2008).

[24] A. R. Calderbank and P. W. Shor, Phys. Rev. A 54, 1098 (1996).

[25] A. Steane, Proc. R. Soc. A 452, 2551 (1996)

[26] L. Isenhower, M. Saffman, and K. Mølmer, Quantum Information Processing 10, 755 (2011).

[27] J. Gulliksen, D. D. B. Rao, and K. Mølmer, EPJ Quantum Technology 2, 1 (2015).

[28] T. Monz, K. Kim, W. Hänsel, M. Riebe, A. S. Villar, P. Schindler, M. Chwalla, M. Hennrich, and R. Blatt, Phys. Rev. Lett. 102, 040501 (2009).

[29] A. Fedorov, L. Steffen, M. Baur, M. P. da Silva, and A. Wallraff, Nature 481, 170 (2012)

[30] S. Aaronson and D. Gottesman, Phys. Rev. A 70, 052328 (2004).

[31] S. Bravyi A. and Aargo, Phys. Rev. A 88, 062308 (2013).

[32] D. S. Wang, A. G. Fowler, A. M. Stephens, and L. C. L. Hollenberg, Quantum Info. Comput. 10, 456 (2010).

[33] A. W. Cross, D. P. Divincenzo, and B. M. Terhal, Quantum Info. Comput. 9, 541 (2009).

[34] Y. C. Cheng and R. J. Silbey, Phys. Rev. A 72, 012320 (2005).

[35] L. S. Theis, F. Motzoi, F. K. Wilhelm, and M. Saffman, "A high fidelity rydberg blockade entangling gate using shaped, analytic pulses," (2016), arXiv:1605.08891.

\section{SUPPLEMENTARY MATERIAL}

\section{Additional details for the Steane code}

Here we provide further detail on the Steane code circuit, and the additional stabilizer information in particular. Since much of this discussion is relevant to measurement-based error correction, we will begin with that case before proceeding to the measurement-free case. The Steane code requires 7 data qubits, and has 6 stabilizer generators, encoding a single logical qubit. The $X$ and $Z$ logical operators are $X_{L}=X^{\otimes 7}$ and $Z_{L}=Z^{\otimes 7}$. The bit-flip and phase-flip errors can be treated independently in the Steane code, so we will restrict ourselves to the discussion of bit-flip errors.

Here, to emphasize the symmetry of the Steane code, we adopt a different labelling convention for the stabilizers. For bit-flip errors, we will take the $3 Z$-type stabilizers to be

$$
\begin{aligned}
& S_{1}=Z_{1} Z_{2} Z_{3} Z_{7}, \\
& S_{2}=Z_{1} Z_{2} Z_{4} Z_{6}, \\
& S_{3}=Z_{1} Z_{3} Z_{4} Z_{5} .
\end{aligned}
$$

Since these all commute, by taking all possible products we can form 7 stabilizer operators. All of these operators stabilize the logical subspace. To understand the utility of considering the additional stabilizers, it is helpful to examine which stabilizers act on which qubits, and which qubits affect which stabilizers. This is captured in Fig. 5. From the diagram, we can point out several convenient features of the enlarged stabilizer set. In particular, note that each stabilizer acts on exactly four qubits. Also, each qubit affects the value of exactly four stabilizer values. In fact, swapping the rows and columns of Fig. 5 produces an identical structure. 


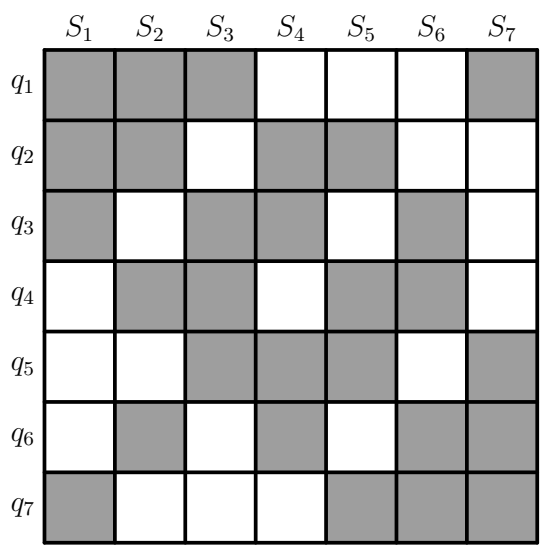

FIG. 5. The qubit-stabilizer structure, where a grey box indicates that the corresponding stabilizer (column) acts on the corresponding qubit (row). For $Z$-type stabilizers, e.g., $S_{1}=Z_{1} Z_{2} Z_{3} Z_{7}$. Thinking of the columns as ancillas, note that a single error on a data qubit would leave exactly four ancillas in the $|1\rangle$ state.

While considering only the stabilizer generators, an error on say $q_{7}$, will affect only a single stabilizer value $\left(S_{1}\right)$, while an error on $q_{1}$ would affect all three generators. By considering the full set we see that all qubits are on equal footing, so that without loss of generality we can examine errors on a single data qubit, knowing that the results will generalize to other qubits.

Thus, examining $q_{1}$ in Fig. 5, it is evident that any two stabilizers overlap at exactly 2 sites. For example, $S_{1}$ and $S_{2}$ both act on $q_{1}$ and $q_{2}$. This overlap property is a powerful feature of the enlarged stabilizer set, particularly in the context of measurement-based error correction.

The syndrome extraction procedure encodes the value of each stabilizer onto an ancilla qubit, requiring 14 total qubits. Here, we will label the ancillas and the stabilizers using the same symbols. If a data qubit experiences an error, then a properly extracted syndrome will leave exactly 4 ancilla qubits in the $|1\rangle$ state. Therefore, if a single extraction error occurs, corrupting a single ancilla, then we will either have 3 or 5 ancilla qubits in the $|1\rangle$ state. Without loss of generality, if $q_{1}$ experiences an error, and the $S_{7}$ value is improperly extracted, then ancilla qubits $S_{1}$, $S_{2}$, and $S_{3}$ will be in the $|1\rangle$ state. However, $q_{1}$ is the only qubit that would have caused all three of those stabilizers to be incorrectly extracted, so we deduce that $q_{1}$ experienced a data error.

Similarly, if 5 ancillas occupy the $|1\rangle$ state, it remains possible to uniquely identify a data qubit. Continuing with our example data error on $q_{1}$, suppose that in addition to the usual four stabilizers, we measure $S_{4}$ in the $|1\rangle$ state. Now, for qubits $q_{2}$ and $q_{3}$, we will measure 3 stabilizers indicating that they had an error. However, all 4 of the $q_{1}$ stabilizers indicate error. Thus, we again correctly identify the data error. We emphasize again that our example generalizes to all qubits and stabilizers. That is, no single encoding error will cause error correction to fail.

Lastly, if two ancilla qubits are corrupted during extraction, we will either measure 2,4 , or 6 ancillas in the $|1\rangle$ state. Clearly, we can identify 2 and $6|1\rangle$ stabilizers as a nonsensical syndrome. Since every pair of qubits overlaps at exactly two stabilizers, there is no way to turn a properly extracted syndrome for one qubit into a properly extracted syndrome for another qubit. Thus, we again detect a nonsensical syndrome and deduce that 2 extraction errors occurred. In this case, this syndrome is simply discarded and extracted again.

Thus, the combination of syndrome extraction and syndrome matching is automatically robust. There is no need for ancilla verification. Furthermore, we do not need to directly entangle the ancillas with each other, and they are immune to phase errors.

For the measurement-free case, some benefits of the additional stabilizers are lost. In particular, in the measurementbased context, it is fairly straightforward to interpret syndromes with extraction errors. However, losing measurement as a resource leaves two options. On one hand, a sufficiently complicated classical logic circuit (on quantum hardware) could properly interpret a wide range of syndromes without measurement. However, we opted for an approach based on making the circuit as simple as possible, with the cost of losing some robustness of the syndrome matching.

Our procedure only corrects data errors if a syndrome is properly extracted. In the event of a faulty extraction, data qubits remain unaffected. This means that preexisting data errors can possibly survive a single error correction cycle, but our circuit prevents further harm from propagating to the data. Note that this is still sufficient for fault-tolerance, since any single-qubit error at any point in the circuit will cause logical failure.

To do this, we use $\mathrm{C}_{4} \mathrm{NOT}$ gates to correct errors on the data qubits. The syndrome is extracted onto seven 
ancillary qubits as in the measurement-based case. Then, the four ancillas corresponding to each data qubit serve as the controls in the $\mathrm{C}_{4} \mathrm{NOT}$ gates. The full circuit is shown in Fig. 2.

\section{Additional details for the Bacon-Shor code}

The procedure for the Bacon-Shor code is quite similar to the bit-flip code. To see this, recall that the bit-flip code is comprised of logical states $|000\rangle$ and $|111\rangle$. An analogous 3 -qubit phase flip code would use logical states $|+++\rangle$ and $|---\rangle$. Note that the bit-flip code, if concatenated, would require 9 data qubits. For the Bacon-Shor code, we "concatenate" a bit-flip code with a phase-flip code. The result is a 9 qubit code that corrects arbitrary single-qubit errors. Restricting to bit-flip errors, the Bacon-Shor stabilizer structure is identical to that of the bit-flip code. So we can apply the same method as in the bit-flip code.

For bit-flip errors, and picturing the data qubits in a $3 \times 3$ array, we have stabilizers

$$
Z_{U}=\left(\begin{array}{lll}
Z & Z & Z \\
Z & Z & Z \\
I & I & I
\end{array}\right)
$$

and

$$
Z_{D}=\left(\begin{array}{lll}
I & I & I \\
Z & Z & Z \\
Z & Z & Z
\end{array}\right)
$$

Note that two $X$ operators acting in the same row commute with these stabilizers, as well as the logical operators $Z_{L}=Z^{\otimes 9}$ and $X_{L}=X^{\otimes 9}$. Thus, if a bit-flip error occurs in one row, we can correct it by applying an $X$ gate to any qubit in the same row. With this in mind, we proceed as with the bit-flip code. We define $Z_{M}=Z_{U} \cdot Z_{D}$ and extract all three stabilizer values. Then, errors are corrected row by row, as opposed to qubit by qubit in the 3-qubit case. The circuit is shown in Fig. 6]

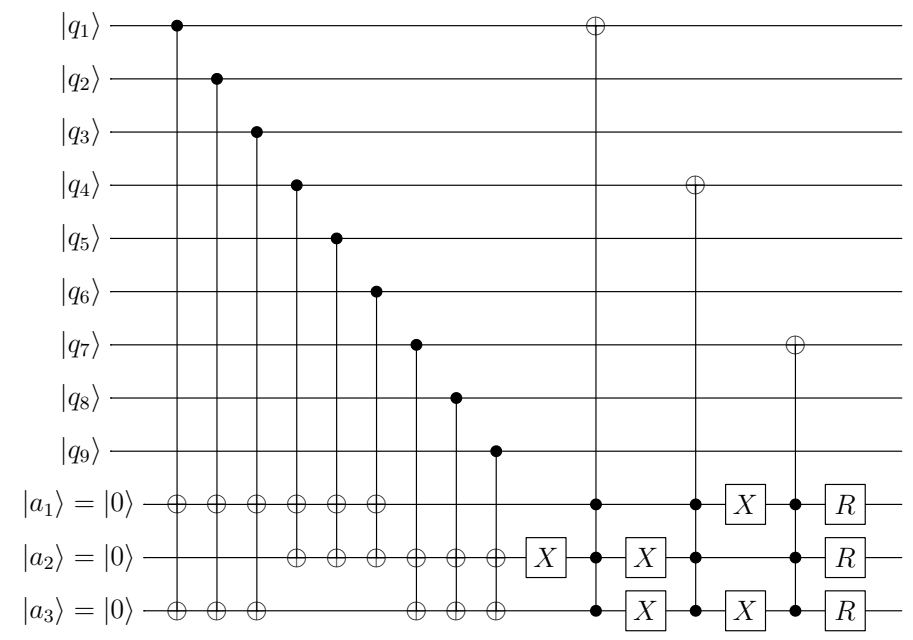

FIG. 6. The full bit-flip error extraction and correction circuit for the BS code. The first 9 gates are for syndrome extraction, the $\mathrm{C}_{3} \mathrm{NOT}$ gates correct the errors. Phase errors are corrected in an analogous process, but data qubits are grouped by columns instead of rows. Here, the rows are (123), (456), and (789).

\section{Simulation details}

The error model introduces possible memory errors at every time step, and for every qubit. Thus, there are a total of $q t$ memory error sites, for $q$ total qubits and $t$ time steps. Similarly, there are a total of $g$ gate error sites. Each 
of these sites is affected by an error with a fixed probability, so a sequence with $i$ memory errors and $j$ gate errors occurs with probability

$$
P(i, j)=\left(\begin{array}{c}
q t \\
i
\end{array}\right)\left(\begin{array}{c}
g \\
j
\end{array}\right) p_{\text {mem }}^{i}\left(1-p_{\text {mem }}\right)^{q t-i} p_{\text {gate }}^{j}\left(1-p_{\text {gate }}\right)^{g-j}
$$

Here, we combine two previous simulation techniques. Note that the entire error correction procedure is Markovian. Thus, we can determine transfer rates between logical states and use these transfer rates to extract information about logical failure rates [31]. Rather than tracking all possible states, we consider our logical states as either being logically correct, correctable, or failed. The states with correctable errors can be further split into single bit-flip, single phaseflip, and both types of error at once. Thus, we must determine the transition probabilities between these 5 subclasses of logical states. We assume that once a state has experienced a logical failure, it will never accidentally correct itself.

To determine the transfer rates between two logical states of type $a$ and $b$, we used a combinatorial expansion, similar to [32]. There, the expansion was used to calculate failure probabilities directly, but we use it here to find the transition rates. That is,

$$
T_{a b}=\sum_{i, j} \alpha_{a b} P(i, j)
$$

We determine the $\alpha_{a b}$ by sampling over the appropriate fault paths and input states. Note that $P(i, j)$ shrinks rapidly for small error rates, so the sum can be truncated at low order. Using the computed transfer matrix, it is possible to examine the system dynamics over repeated error correction cycles.

For a single uncorrected qubit with error rate $p$, the chance that a logical failure has occurred grows as $\sum_{i=0}^{T} p \cdot(1-p)^{i}$. By comparing logical failure rates with growth of this form, we can extract a logical error rate $p_{L}$. 